\title{
Immiscible-fluid displacement: Contact-line dynamics and the velocity-dependent capillary pressure
}

\author{
Ping Sheng and Minyao Zhou \\ Exxon Research and Engineering Company, Route 22 East, Clinton Township, Annandale, New Jersey 08801 \\ (Received 8 October 1990; revised manuscript received 29 March 1991)
}

\begin{abstract}
The dynamics of immiscible-fluid displacement is studied in the simple geometry of a capillary tube. Here the interesting physics lies in the breakdown of the no-slip boundary condition near the contact line, defined as the intersection of the fluid-fluid interface with the solid wall. Through numerical hydrodynamic calculations, we link macroscopic-flow behavior to the microscopic parameters governing the contact-line region. It is shown that the moving contact line generates two types of frictional forces. One, the viscous stress, is responsible for the observed deformation of the fluid-fluid interface as the flow velocity $U$ increases. Our calculation is in excellent agreement with prior analytic works on this aspect. In particular, our results reproduce Hoffman's scaling relation as well as the logarithmic dependence of the viscous friction on slipping length. Identical macroscopic-flow behaviors are also found to result from three different slipping models provided that their slipping lengths are each renormalized by a model-dependent constant. Besides the viscous stress, however, comparison with experiments revealed a second frictional force that varies as $U^{x}$, with $0<x \leq 0.5$, which is dominant at capillary numbers $<10^{-3}$. We propose that the source of this new friction is the excitation of damped capillary waves at the fluid-fluid interface due to contact-line motion over wall roughness. Consideration of this mechanism yields not only the correct range of $x$ values, but also good agreement with the measured magnitude of the second frictional force. The paper concludes with an analysis of the frequency-dependent pressure response to an imposed ac velocity perturbation. An expression is derived for the critical frequency that separates the low-frequency behavior from that of the high-frequency regime.
\end{abstract}

PACS number(s): 47.55.Kf, 03.40.Gc, 68.10. $-\mathrm{m}, 68.45 .-\mathrm{v}$

\section{INTRODUCTION}

Immiscible-fluid displacement is a common phenomenon whose diverse ramifications have been the subjects of continued research over the past few decades. In Hele-Shaw cells or porous media, the displacement is known to result in unstable fingering patterns, some of which characterizable as fractals, whose dependences on the relative viscosity, wetting property, velocity of displacement, and other physical parameters have only recently begun to be understood. Apart from the patternformation aspect of the phenomenon, but intimately related to it at the fundamental level, is the problem of the moving contact line [1], defined as the intersection of the fluid-fluid interface with the solid wall. Here the problem in its simplest form may be stated as follows. When one fluid displaces another immiscible fluid, the contact line appears to "slip" relative to the solid wall, in direct violation of the traditional no-slip boundary condition at the fluid-solid interface.

There have been two resolutions of this classical problem depending on the wetting property of the fluids. When one of the fluids completely wets the solid surface, or when the contact angle between the fluid-fluid interface and the solid wall is small, it was proposed that a thin "precursor film" of the wetting fluid actually precedes the fluid-fluid interface so that there is no true contact line [2]. On the other hand, when the fluids only partially wet the solid it was initially proposed that the noslip boundary condition can be maintained by the fluids "rolling" over the solid wall in much the same manner as a bicycle tire rolls over the ground (without slipping) such that the point of tread contact only appears to "slip" forward [3]. Analytical solution to the problem can be written down following this physical picture, but it was discovered that the calculated viscous stress diverges at the contact lines as $1 / z$ for any finite velocity, where $z$ is the distance on the wall away from the contact line. The existence of this divergence is understandable since the fluids have to execute infinitely sharp turns at the contact line. However, what makes this solution unphysical is the fact that the divergence is nonintegrable (since the integral of $1 / z$ yields $\ln z$ ), implying infinite dissipation. This is to be contrasted with the physical divergence of the electric field $|\mathbf{E}|$ at a sharp point, where the integral of $|\mathbf{E}|^{2}$ always converges. The nonintegrable divergence therefore directly implies (in the partial wetting case) the breakdown of the no-slip boundary condition, at least in a region close to the contact line. What may first "appear" as slipping ends up, after much analysis, as actual slipping. Recently, this slipping picture was supported by results of moleculardynamics simulations $[4,5]$, which explicitly demonstrated the slipping of the moving contact line. Slipping thus removes the unphysical singularity but makes the resulting macroscopic behavior of immiscible-fluid displacement directly dependent on the microscopic parameters governing the region close to the contact line.

Our motivation for this work is to use first-principle hydrodynamic calculations to relate the microscopic pa- 
rameters governing the slipping region directly to macroscopic-flow behavior for immiscible fluids in a capillary tube. By measuring macroscopic flow parameters, it was initially expected (maybe naively) that one can use the theory to obtain information about the slipping region. Our calculations indeed support the conclusions of prior studies [1] on some aspects of the macroscopicflow behavior, such as the manner in which the viscous deformation of the fluid-fluid interface increases as the velocity $U$ is increased. Yet quantitative comparison with experiments revealed a second frictional force [6] at low velocities that varies as $U^{x}$, where $0<x<0.5$, which is not accounted for by prior theories. We propose that the source of this new frictional force is the excitation of damped capillary waves at the fluid-fluid interface due to contact-line motion over wall roughness. Consideration of this mechanism yields excellent agreement with experimental data.

In what ensues, slipping models and formulation of the calculational approach are given in Sec. II. Viscousstress effects are described in Sec. III, followed by a comparison with experiments and a discussion of the new frictional force and its generating mechanism in Sec. IV. In Sec. $\mathrm{V}$ we consider the frequency modulation aspects of the interfacial dynamics. The paper concludes with a summary and remarks about the potential direction of future studies in Sec. VI.

\section{SLIPPING MODELS AND FORMULATION OF THE CALCULATIONAL APPROACH}

\section{A. Slipping models}

Consider two immiscible fluids labeled 1 and 2 in a capillary tube of radius $R$, assumed to be small enough so that the gravity effects may be neglected. At rest, the interface between the two fluids forms a static contact angle $\theta_{0}$ with the wall as shown in Fig. 1 . The contact line in this case is a circle defined by the intersection of the fluid-fluid interface with the tube wall. As a result of the capillary pressure, it is necessary to have a counter-balancing pressure difference of $p_{0}=p_{1}-p_{2}$ $=-2 \gamma \cos \theta_{0} / R$ to maintain the static state. Here $\gamma$ is

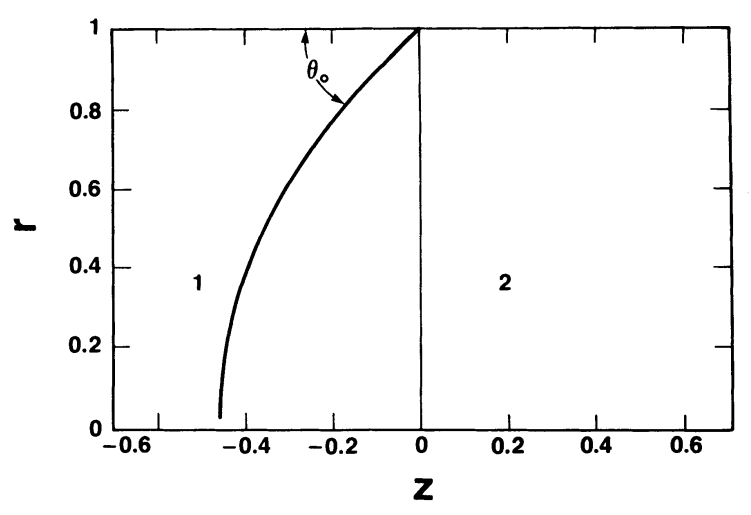

FIG. 1. Static interface $Z_{0}(r)$ between fluid 1 and fluid 2 . the surface tension of the fluid-fluid interface. In this paper, all calculations will be performed in the comoving frame in which the interface is stationary. Any pressure difference in excess of $p_{0}$ is counterbalanced by the viscous pull of the moving tube wall.

During the fluid-displacement process the manner in which the slipping occurs at the contact line is not known in detail at present. Here we consider three slipping models. In model $\mathrm{I}$ we let $v_{z}=0$ at the slipping contact line, $z=0$, and $v_{z}=-U\left[1-\exp \left(-|z| / l_{s}\right)\right]$ away from the contact line [6], where $l_{s}$ denotes the slipping length. In model II we adopt the classical Navier slipping model [1] where the relative slipping velocity, $v_{z}+U$, is proportional to the tangential stress $\sigma_{z r}$ at the wall, i.e., $v_{z}+U=\left(l_{s} / \eta\right) \sigma_{z r}$, with $\eta$ denoting the fluid viscosity. In model III we consider slipping the model.of Huh and Mason [7], where $\sigma_{z r}=0$ at $|z| \leq l_{s}$, with the no-slip boundary condition operative at $|z|>l_{s}$. In all three cases $l_{s}$ is the model parameter whose value has a direct bearing on the macroscopic immiscible-flow behavior.

It should be noted that the three models above are all phenomenological in nature. On the microscopic scale, there are two possible ways in which slipping can occur. In the simple case of a solid wall smooth on the molecular scale, molecular-dynamics simulations have shown $l_{s}$ to be on the order of $10 \AA[4,5]$. On the other hand, for the more realistic case of a rough wall, the same phenomenological effect of slipping can occur when the contact line "jumps" across indentations. Jansons [8] has shown that such jumps can give rise to the same macroscopic behaviors as slipping does, but with a slipping length that is inversely proportional to $\mathrm{Ca}$, i.e., $l_{s}=l_{d} / \mathrm{Ca}$, where $l_{d}$ denotes the scale of wall roughness. However, physically the jump process is very different from the smooth wall case since the jerky motion of the contact line (resulting from the jumps) introduces a nearly periodic time dependence into the problem. The phenomenological models, which are all time independent, are recovered only in a time-averaged sense. Furthermore, there are two ways in which a "jump" can occur. In one scenario, proposed by Joanny and Robbins [9], the contact line is assumed to move very quickly through a trough, thus simulating a jump. In the other case, Jansons's picture is that the contact line can encounter points on the rough surface where the local force balance no longer holds, and the contact line jumps with capillary number $\mathrm{Ca} \sim 1$ (slowed down only by the viscosity of the fluid). Whereas in the first scenario the slipping length is still $\sim 10 \AA$, in the second case the slipping length can be on the order of the wall roughness and larger [8]. In any case, it is probable that whereas the picture of Joanny and Robbins is valid when the amplitude of the roughness is small; jumps in the sense of Jansons can occur when the ratio between roughness amplitude and $l_{d}$ becomes large. Details of our model can be found in Sec. IV B.

The near-periodic time dependence of the contact-line motion in the rough-wall case can give rise to additional capillary-wave excitations at the fluid-fluid interface. The effect of these excitations will be considered in Sec. IV. 


\section{B. Calculational approach}

Given a slipping model, the calculational task is to solve the nondimensionalized time-independent NavierStokes equation with the incompressibility condition

$$
\begin{aligned}
& -\nabla p+\mathrm{Ca}^{2} \mathbf{v}=(\mathrm{Ca})(\mathrm{Re}) \mathbf{v} \cdot \boldsymbol{\nabla} \mathbf{v}, \\
& \boldsymbol{\nabla} \cdot \mathbf{v}=0 .
\end{aligned}
$$

Here $v$ is measured in units of $U$, pressure $p$ is in units of $\gamma / R$, and length is in units of $R$. Fluid density is denoted by $\rho$. The capillary number $\mathrm{Ca}=\eta U / \gamma$ measures the ratio of viscous stress to surface tension, and the Reynold's number $\operatorname{Re}=\rho U R / \eta$ measures the ratio between inertial effect and viscous stress. In our calculations the nonlinear term $\mathbf{v} \cdot \nabla \mathbf{v}$ shows negligible effects for all the cases addressed, since $\mathrm{Re} \lesssim 1$ for $\mathrm{Ca} \lesssim 10^{-2}$ and the experimental values of $\rho, R$, and $\eta$. Therefore for all practical purposes the nonlinear term may be set equal to zero. The capillary number $\mathrm{Ca}$, which may be regarded as the dimensionless velocity, is thus the only controlling parameter in Eq. (1).

For the boundary conditions, at the tube wall $(r=1)$ we use the slipping models specified above for $v_{z}$, and let $v_{r}=0$. Far from the interface viscous damping should make the flow insensitive to what happens near the interface, and thus Poiseuille-like. That means at $z= \pm L$, where we let $L=5, \partial v_{z} / \partial_{z}=0, v_{r}=0$, and $p=$ const. The choice of $L=5$ has been checked to be adequate since letting $L=6,7$ produces no discernible changes in the solution. At the fluid-fluid interface, we must have $v_{1}=v_{2}$, $\mathbf{v} \cdot \hat{\mathbf{n}}=0$, where $\hat{\mathbf{n}}$ is the interface unit normal, and stress balance

$$
\begin{aligned}
& \left(p_{1}-p_{2}\right) \hat{\mathbf{n}}=\left(\sigma^{(1)}-\sigma^{(2)}\right) \cdot \hat{\mathbf{n}}+2 \kappa \hat{\mathbf{n}}, \\
& \sigma_{z z}=2 \mathrm{Ca} \frac{\partial v_{z}}{\partial z}, \\
& \sigma_{z r}=\mathrm{Ca}\left(\frac{\partial v_{z}}{\partial r}+\frac{\partial v_{r}}{\partial z}\right), \\
& \sigma_{r r}=2 \mathrm{Ca} \frac{\partial v_{r}}{\partial r},
\end{aligned}
$$

where $\sigma^{(i)}$ denotes the stress tensor of the fluid $i$ expressed in units of $\gamma / R$, and $\kappa$ (in units of $R^{-1}$ ) is the principal curvature of the interface $Z_{0}(r)$, which must also satisfy the requirements that at $r=1$ it forms an angle $\theta_{0}$ with the wall, and $d Z_{0}(r) / d r=0$ at $r=0$.

Due to the unknown interfacial shape $Z_{0}(r)$, which must be obtained self-consistently as part of the solution, the present problem is highly nonlinear. Added difficulty in numerical calculation arises from the small grid size needed to resolve the microscopic-flow behavior inside the slipping region, $|z|<l_{s}$. If one uses a uniform grid size that is small enough to resolve the slipping region, then the number of grid points would be astronomical, certainly beyond the computational power of any existing supercomputer. To make the calculation feasible one must therefore use a nonuniform coordinate system where the grid size is small close to the contact line and gradually increasing in size away from it. We have chosen a hyperbolic-tangent coordinate transformation such that the interface $Z_{0}(r)$, as well as the extra-fine grid points for $|1-r|,|z|<l_{s}$, is transformed to a regular Cartesian coordinate. In particular, the transformation of $(z, r)$ to the Cartesian coordinate system $(X, Y)$ is accomplished by the following:

$$
\begin{aligned}
& z=-Z_{0}(r)+\left[L+Z_{0}(r)\right]\left(1-\frac{\tanh \left(G \frac{L+X}{L}\right)}{\tanh G}\right) \\
& r=\frac{\tanh \left(G Y^{\delta}\right)}{\tanh G}
\end{aligned}
$$

where $G$ and $\delta$ are the two transformation parameters whose values are chosen to optimize the placing of grid points in the $(z, r)$ coordinate system within the constraint of given computed memory (in this case four mega-words of Cray-XMP). It should be noted that the interface $Z_{0}(r)$ is part of the transformation. $Z_{0}(r)$ is transformed to the $Y$ axis of the Cartesian coordinate system.

We use the method of finite differences to carry out the numerical solution of Eqs. (1) and (2) in the transformed $(X, Y)$ coordinate system, with the initial assumptions of a certain pressure difference and a spherical shape for $Z_{0}(r)$. The calculational steps are as follows.

(1) In regions away from the interface, the relevant equations are Eqs. (1a) and (1b), and the variables at each grid point are $\mathbf{v}$ and $p$. At the interface, we have the additional Eqs. (2a) and (2b), with $\kappa$ as the additional variable at each interface grid point.

(2) Once the linear system is solved, a cubic-spline fit of $\kappa(r)$ is obtained, and from $\kappa(r)$ a new $Z_{0}(r)$ is calculated by integrating the following second-order nonlinear differential equation relating the curvature to the surface shape $Z_{0}$ :

$$
\kappa(r)=\frac{1}{2} \frac{Z_{0}^{\prime \prime}+Z_{0}^{\prime \prime}\left[1+\left(Z_{0}^{\prime}\right)^{2}\right] / r}{\left[1+\left(Z_{0}^{\prime}\right)^{2}\right]^{3 / 2}},
$$

with the initial conditions of $Z_{0}=0, Z_{0}^{\prime}=-\left(\tan \theta_{0}\right)^{-1}$ at $r=1$.

(3) Once $Z_{0}(r)$ is integrated to $r=0$, the slope $Z_{0}^{\prime}(0)$ is checked. If it is not zero, then the initially assumed pressure difference is modified and the calculation (including the coordinate transformation) is iterated by using Newton's method until consistency, in the sense of $Z_{0}^{\prime}(0)=0$ and the assumed $Z_{0}(r)$ equals the $Z_{0}(r)$ obtained, is achieved.

(4) The results obtained at each $U$ are used as the initial guess for the solution at a (subsequent) higher $U$.

In this manner the interfacial shape $Z_{0}(r)$, the pressure drop, and the flow patterns are obtained as a function of $U$. Zero-sum checks, such as force balance and entropy generation minus the work done, show that our solution is accurate to $\sim 1 \%$ at $\mathrm{Ca}<10^{-3}$ and $\sim 5-10 \%$ at $\mathrm{Ca} \simeq 10^{-2}$, provided the slipping region is resolved by 
5-10 grid points. In what follows, we present results of calculations with $\eta_{1}=\eta_{2}$ (equal viscosity for fluids 1 and 2). In all the calculations the two microscopic input parameters are $l_{s}$ and $\theta_{0}$. The relationship between $l_{s}$ and $\theta_{0}$ and the macroscopic-flow behavior is the topic of the next two sections.

\section{VISCOUS STRESS EFFECTS}

\section{A. Flow pattern and the apparent contact angle}

In Fig. 2 we show the calculated flow diagram for the low-velocity case of $\mathrm{Ca} \simeq 10^{-3}, l_{s}=6 \times 10^{-3}$, and $\theta_{0}=40^{\circ}$. The slipping model used is that of Huh and Mason [7] (model III), where the tangential stress at $r=1$ inside the slipping region $|z|<l_{s}$ is set equal to zero. On the macroscopic scale, the flow lines exhibit the pattern expected from the existence of a stationary fluid-fluid interface in the comoving frame. This flow pattern remains qualitatively similar for all three slipping models. However, in the slipping region close to the contact line the different models exhibit different behaviors. For model III, shown in Fig. 2, we see two eddies: one on the fluid 1 side of the slipping region, and the other (the narrow one) on the fluid 2 side of the slipping region. For the other two models, due to the fact that the boundary condition imposed by models I and II is a smooth function of $z$, the eddy on the fluid 1 side extends outside the region of $-l_{s} \leq z \leq 0$ for these two cases. Calculated values of pressure and viscous stress all show a dramatic surge in the slipping region (they would diverge if not for the slipping condition inside the slipping region), and their net effects on the interfacial shape are summarized in Fig. 3. Here $l_{s}=10^{-4}, \theta_{0}=40^{\circ}$, and the slipping model $I$ is used. It is seen that as the flow velocity increases, the interface is incrementally deformed to the right until it completely flips to the other side. What should be noted, however, is that $\theta_{0}$ remains at $40^{\circ}$ for all the calculations. The deformed interface is therefore measured by an apparent contact angle

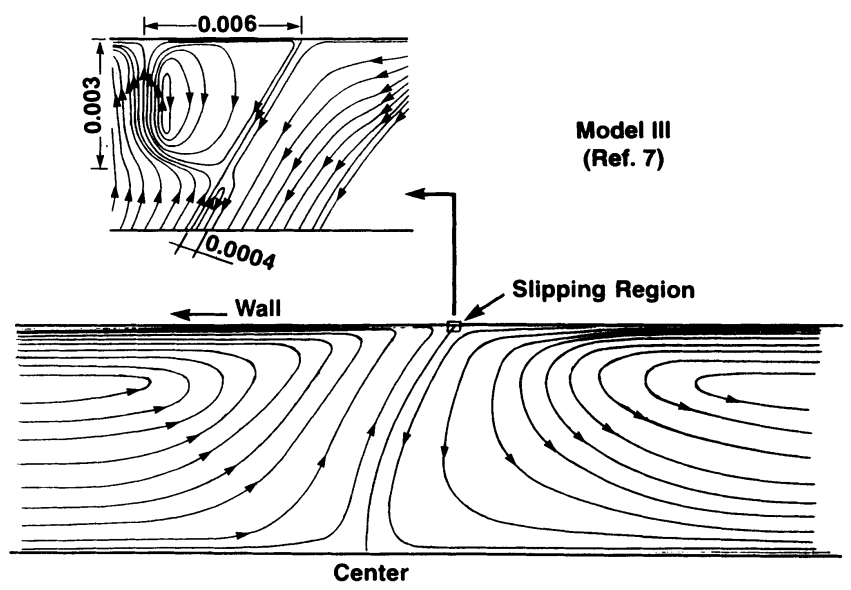

FIG. 2. Flow diagram for $\mathrm{Ca}=10^{-3}, l_{s}=6 \times 10^{-3} R$, and $\theta_{0}=40^{\circ}$. The slipping model of Huh and Mason (model III) was used. The inset shows the slipping region. The left side is fluid 1 ; the right side is fluid 2 .

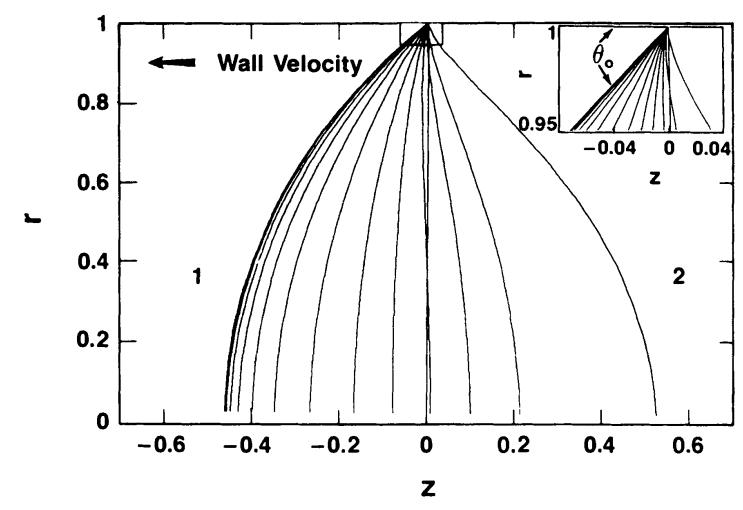

FIG. 3. Interfacial shape $Z_{0}(r)$ calculated with model I, $\eta_{2} / \eta_{1}=1, l_{s}=10^{-4}$, and $\theta_{0}=40^{\circ}$. The leftmost profile is the static one. As $\mathrm{Ca}$ is increased from 0 to $10^{-4}, 2 \times 10^{-4}, 4 \times 10^{4}$, $8 \times 10^{-4}, \quad 1.6 \times 10^{-3}, \quad 6.4 \times 10^{-3}, 1.14 \times 10^{-2}, 1.64 \times 10^{-2}$, $2.14 \times 10^{-2}, 2.64 \times 10^{-2}, 3.14 \times 10^{-2}$, and $3.64 \times 10^{-2}$, the interface flips progressively to the right. Inset: An enlarged view of the contact-line regions. The microscopic contact angle $\theta_{0}$ is fixed at $40^{\circ}$.

$$
\theta=\tan ^{-1}\left(\frac{1-h^{2}}{2 h}\right),
$$

where $h$ is the distance of the interface at $r=0$ from the $z=0$ plane ( $z=0$ is defined by the contact line). The inset in Fig. 3 shows a closer view of the contact region. It is seen that the interface bends continuously from $40^{\circ}$ at $r=1$ to the apparent contact angle outside of the slipping region.

The essential physics of the viscous stress effect may be stated as follows. At $\mathrm{Ca} \simeq 10^{-2}$, which is usually reached only at high flow velocities (for $\eta=0.1 \mathrm{P}, \gamma=20 \mathrm{dyn} / \mathrm{cm}$, $\mathrm{Ca}=10^{-2}$ implies $U=2 \mathrm{~cm} / \mathrm{sec}$ ), the viscous stress at the center of the capillary tube is about two orders of magnitude smaller than the capillary pressure (surface tension), so the interface would appear rigid to the fluid. However, near the contact line the near divergence of the viscous stress (the divergence is cutoff by the slipping condition inside $|z|<l_{s}$ ) makes it comparable to the capillary pressure. The interface is therefore deformed by the viscous stress, forcing the apparent contact angle to deviate from $\theta_{0}$. At the macroscopic level, the different slipping models all exhibit qualitatively the same behavior. Below we show that the macroscopic predictions of the three slipping models can be quantitatively superimposed if one renormalizes the slipping length by a constant factor which is particular to each model.

\section{B. Scaling property}

In Fig. 4 we plot the apparent contact angle, calculated with $l_{s}=10^{-4}$ and slipping model $\mathrm{I}$, as a function of $\log (\mathrm{Ca})$ for $\theta_{0}=40^{\circ}, 60^{\circ}, 80^{\circ}$, and $100^{\circ}$. It is seen that the apparent contact angle always increases monotonically as a function of $\mathrm{Ca}$ until at some point the extrapolated curve reaches $180^{\circ}$. (Our calculation always fails, usually in the form of not showing convergence, before $180^{\circ}$ is reached.) Beyond that point instability sets in, and exper- 


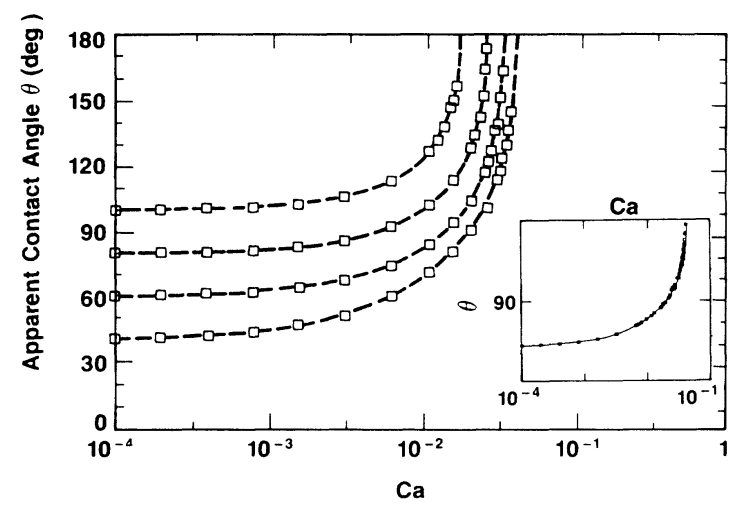

FIG. 4. Apparent contact angle plotted as a function of $\ln \mathrm{Ca}$ for $\theta_{0}=40^{\circ}, 60^{\circ}, 80^{\circ}$, and $100^{\circ}$. The inset shows the scaling of the four curves. The solid line is the fit produced by using Eqs. (6) and (7) with $K=0.3$.

imentally one can have a continuous film of fluid 2 left behind and pinched-off droplets of fluid 1 traveling ahead of the interface in fluid 2. While not apparent from Fig. 4 , the four curves can actually be scaled to a single curve by using the scaling function found by Hoffman [10],

$$
G(\theta)=G\left(\theta_{0}\right)+\mathrm{Ca} \ln \left(K / l_{s}\right),
$$

where $K$ is a slipping-model-dependent constant. It should be noted that if $l_{s} \simeq l_{d} / \mathrm{Ca}$ for the Jansons's picture, then

$$
G(\theta)=G\left(\theta_{0}\right)+\mathrm{Ca} \ln \left(K / l_{d}\right)+\mathrm{Ca} \ln \mathrm{Ca},
$$

provided $l_{d} / \mathrm{Ca} \lesssim K$ since the viscous effect always makes $\theta \geq \theta_{0}$. That means there is a leveling off of $l_{s}$ at $\mathrm{Ca} \lesssim l_{d} / K$. Equation (6a) implies that if the function $G$ has an inverse, then $\theta$ is a function of only one variable

$$
\xi=G\left(\theta_{0}\right)+\mathrm{Ca} \ln \left(K / l_{s}\right),
$$

i.e., $\theta=G^{-1}(\xi)$. It follows that two distinct $\theta_{0}$ values, $\theta_{0}{ }^{(1)}$ and $\theta_{0}{ }^{(2)}$, can yield the same apparent contact angle $\theta$ at $\mathrm{Ca}$ values that differ by $\left\{G\left[\theta_{0}(1)\right]-G\left[\theta_{0}(2)\right]\right\} /$ $\ln \left(K / l_{s}\right)$, so that they give the same value of $\xi$. Two $\theta$ vs Ca curves, calculated at different $\theta_{0}$ values, may therefore be superimposed upon each other through a uniform additive shift horizontally along the $\mathrm{Ca}$ axis. This is done in the inset to Fig. 4, and we see that the four curves indeed collapse into one (with $\theta_{0}=40^{\circ}$ ). Moreover, we have fitted our numerical data by the solid curve representing Eq. (6a) where the function $G(\theta)$ is given by

$$
\begin{aligned}
& G(\theta)=\int_{0}^{\theta} d \phi[f(\phi)]^{-1}, \\
& f(\phi)=\frac{2 \sin \phi\left\{q^{2}\left(\phi^{2}-\sin ^{2} \phi\right)+2 q\left[\phi(\pi-\phi)+\sin ^{2} \phi\right]+(\pi-\phi)^{2}-\sin ^{2} \phi\right\}}{q\left(\phi^{2}-\sin ^{2} \phi\right)[(\pi-\phi)+\sin \phi \cos \phi]+(\phi-\sin \phi \cos \phi)\left[(\pi-\phi)^{2}-\sin ^{2} \phi\right]},
\end{aligned}
$$

where $q=\eta_{2} / \eta_{1}$. This form of $G(\theta)$ was derived by Cox [11] by the method of matching asymptotic expansions. The excellent fit in this case is obtained by letting $K=0.3$. In fact, for models II and III we get equally excellent fits with $K=0.05$ and 1 , respectively. What this demonstrates is the fact that, in spite of significant microscopic differences, macroscopically the three slipping models all exhibit the same behavior. The only quantitative difference is in the value of $K$. Our numerical results thus completely support prior works [1] on this issue. The excellent agreement of Eqs. (6) and (7) with our numerical data means that, provided the value of $K$ is known, the scaling function can serve as a simpler way to evaluate the $\theta$ vs $\mathrm{Ca}$ behavior.

It should be noted here that for $q=1$ and $|\cos \theta|<0.6$, Eq. (6a) is excellently approximated by

$$
\cos \theta_{0}-\cos \theta \simeq 5.63 \mathrm{Ca} \ln \left(K / l_{s}\right)
$$

Since $\cos \theta$ represents a capillary pressure drop across the interface, the increase of this pressure drop is seen from Eq. (8) as linearly proportional to the velocity, or $\mathrm{Ca}$. This is expected, since viscous stress, which gives rise to the effect of changing $\theta$, is linearly proportional to the velocity.

\section{Pressure drop due to the moving interface}

The other important property of macroscopic fluid flow is the pressure drop-flow rate relationship. By keeping $\theta_{0}$ constant and $\eta_{1}=\eta_{2}$, the total pressure drop, $p_{1}-p_{2}$, can be expressed as the sum of two parts

$$
p_{1}-p_{2}=2 \Delta P+16(\mathrm{Ca}) L,
$$

where $\Delta P$ denotes half the pressure drop arising from the interface, and $16(\mathrm{Ca}) L$ denotes the pressure drop of the single-phase Poiseuille flow, which is linearly proportional to the sample length $L$. From our numerical data, we found

$$
\Delta P \simeq-\cos \theta
$$

In Fig. 5 the calculated $\Delta P$ (in units of $\gamma / R$ ) is plotted versus $\mathrm{Ca}$. On the same graph $-\cos \theta$ is also shown. Comparison shows that $\Delta P$ is slightly smaller than $-\cos \theta$ with the difference increasing (nearly) linearly with $\mathrm{Ca}$. This is due to the fact that $\Delta P$ is obtained from the total calculated pressure drop by subtracting from it the Poiseuille-flow component. However, since the average flow rate in the interface region is considerably lower than that far away from the interface, the Poiseuille com- 


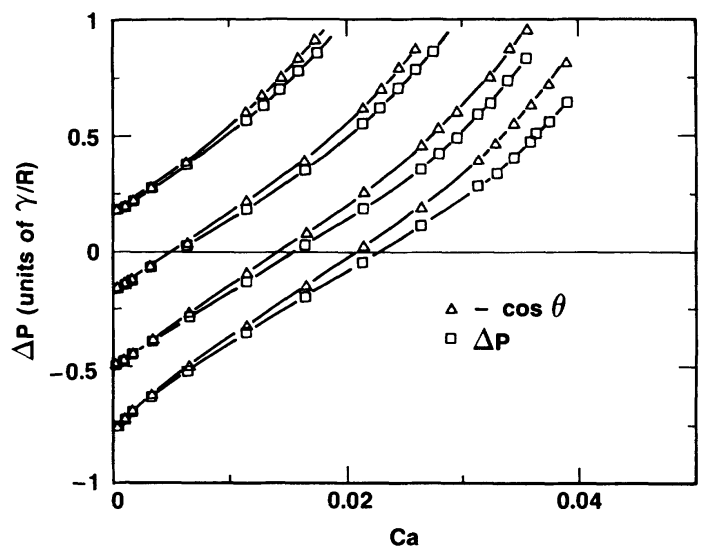

FIG. 5. The pressure drop associated with the interface plotted as a function of $\mathrm{Ca}$, shown by the open squares. In the same graph is also plotted the Ca variation of $-\cos \theta$, shown by the open triangles. Similarity of $\Delta P$ with $-\cos \theta$ is clearly seen.

ponent thus represents an upper bound to that component of the pressure drop.

Since $\Delta P$ is due to the moving interface, it would be interesting to verify its dependence on the two contact line parameters, $\theta_{0}$ and $l_{s}$. Equations (8) and (9b) display that dependence, which states in particular that $\Delta P$ should vary linearly with $-\ln \left(l_{s}\right)$ as a result of the $1 / z$ stress divergence that is made finite by the slipping region. Figure 6 plots $\Delta P$ as a function of $\ln \left(l_{s}\right)$ for a fixed Ca. An excellent linear relation is obtained. The predicted slope of $\left(-5.63 \times 10^{-4}\right) 2.3 \simeq-0.0013$ from Eq. (8) is also in excellent agreement with the measured slope of -0.0014 . The linear relationship between $\Delta P$ and $-\cos \theta_{0}$ may be deduced from Eqs. (8) and (9b).

The fact that $\Delta P \simeq-\cos \theta$ implies that one cannot deduce the values of $\theta_{0}$ and $l_{s}$ from macroscopic measurements [12] of $\theta$ and $\Delta P$ at a given value of Ca. Thus, for example, the same value of apparent contact angle $\theta \simeq 97^{\circ}$ is obtained at $\log _{10} \mathrm{Ca} \cong-1.65$ for two combinations of $l_{s}=10^{-3}, \theta_{0}=60^{\circ}$ and $l_{s}=10^{-4}, \theta_{0}=40^{\circ}$. Can one use the pressure drops to resolve the two cases? We found the total pressure drops in the two cases to be 32.62 and 32.95 , respectively, which are identical to

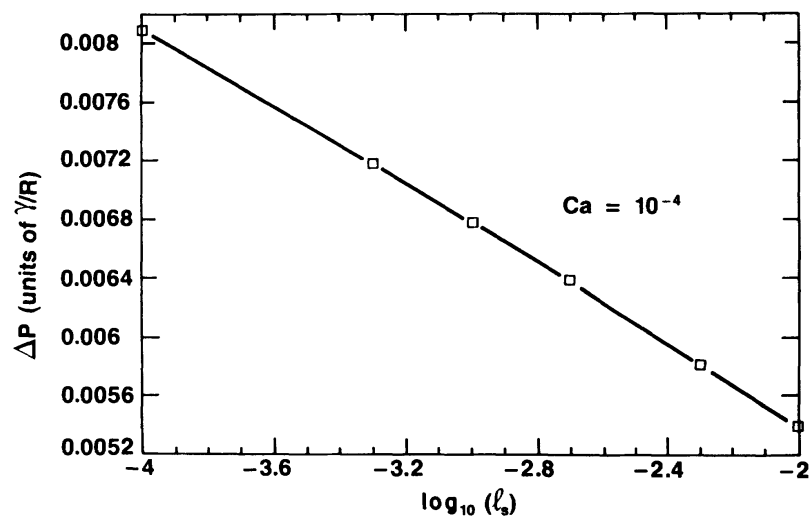

FIG. 6. Interface pressure drop $\Delta P$ plotted as a function of $\log _{10} l_{s}$ for $\mathrm{Ca}=10^{-4}$. Excellent logarithmic variation is seen. within the accuracy of our computation. The reason for both this degeneracy and the fact that different slipping models produce the same macroscopic consequences is that the singularity at the contact line is weak $(\ln z)$, and consequently different microscopic behaviors within the slipping region all result in the same asymptotic behavior outside the slipping region [1]. However, the next section shows that it is still possible to separate out the effect of $l_{s}$ from that of $\theta_{0}$ due to the existence of a second frictional force [6].

\section{COMPARISON WITH EXPERIMENTS AND THE VELOCITY-DEPENDENT CONTACT ANGLE}

\section{A. Microscopic contact angle and the new frictional force}

In the previous section we have seen that the viscous stress is responsible for the apparent contact angle $\theta$ to deviate from $\theta_{0}$. Moreover, for $q=1$ the quantity $\cos \theta_{0}-\cos \theta$ is (approximately) linearly proportional to $\mathrm{Ca}$. Is that valid experimentally? Figure 7(a) shows the apparent contact angle data by Fermigier and Jenffer [13]. It is seen that the experimental results have the qualitative behavior as predicted. This is expected, since the interface is always observed to curve more and more towards the direction of motion as $\mathrm{Ca}$ increases. The solid curves in Fig. 7(a) are drawn by using Eqs. (6a) and (7) with $K=0.3$, the parameter values of $\theta_{0}=50^{\circ}, 95^{\circ}$, and $117^{\circ}$, and values of $l_{s}=10-10^{-11} \AA$. The physically unreasonable values of $l_{s}$, those less than $1 \AA$, are required in order to reproduce the $\mathrm{Ca}$ value where the apparent contact angle $\theta$ reaches $180^{\circ}$. Recently, Thompson and Robbins [14], using molecular-dynamics simulations, have shown that under certain assumptions $\ln K$ can be enhanced by large factors so that one may not require $l_{s}$ to be unphysically small. However, even if this were the case, the solid lines in Fig. 7(a) clearly show that at small $\mathrm{Ca}$ there still exist significant discrepancies between the prediction of Eq. (6a) [the use of Eq. (6b) does not change the conclusion] and the experimental data. The essential point here is that if we consider only viscous stress effects, which is given by Eq. (6), the apparent contact angle $\theta$ can only deviate from $\theta_{0}$ by an insignificant amount at $\mathrm{Ca}<10^{-2.7}$, even if we allow $K$ to be significantly enhanced. Therefore, any significant variation of $\theta$ observed at $\mathrm{Ca}<10^{-2.7}$ may be attributed to a velocity-dependent $\theta_{0}$.

The microscopic contact angle $\theta_{0}$ is determined by the force balance at the contact line, i.e.,

$$
\cos \theta_{0}=\frac{\Delta \alpha}{\gamma},
$$

where $\Delta \alpha$ denotes the difference in the interfacial energies between the wall and the two fluids. For $\theta_{0}$ to vary as a function of velocity, there must be a velocitydependent frictional force $F$ such that

$$
\cos \theta_{0}(0)-\cos \theta_{0}(\mathrm{Ca})=\frac{F(\mathrm{Ca})}{\gamma},
$$

with $F(0)=0$. To analyze the data shown in Fig. $7($ a) for the dependence on $\mathrm{Ca}$, Fermigier [15] has replotted the 
data in the log-log scale and concluded that if there is a dependence of $\theta(U)-\theta(0)$ on $\mathrm{Ca}$ in the form of $\mathrm{Ca}^{x}$, then $x>0.5$. Mumley, Radke, and Williams [16] have also carried out a similar analysis on their data and concluded that $x \simeq \frac{1}{2}$. However, these analyses did not look at $\cos \theta_{0}(\mathrm{Ca})$. Instead, they plotted the dependence of the apparent contact angles $\theta$ on $\mathrm{Ca}$. As we have seen, $\cos \theta$ has an intrinsic $x=1$ dependence that becomes dominant as $\mathrm{Ca}$ increases. Since the data shown in Fig. 7(a) have many points with $\mathrm{Ca}>10^{-3}$, it is clear that in order to look at the intrinsic $\mathrm{Ca}$ dependence of $\cos \theta_{0}$, one should substract off the linear $\mathrm{Ca}$ dependence of the viscousstress effect from the data, i.e., $\theta_{0}$ should be obtained from the data by

$\theta_{0}= \begin{cases}g^{-1}\left[g(\theta)-\mathrm{Ca} \ln \left(K / l_{d}\right)-\mathrm{Ca} \ln \mathrm{Ca}\right], & \mathrm{Ca} \geq l_{d} / K \\ \theta, & \text { otherwise }\end{cases}$

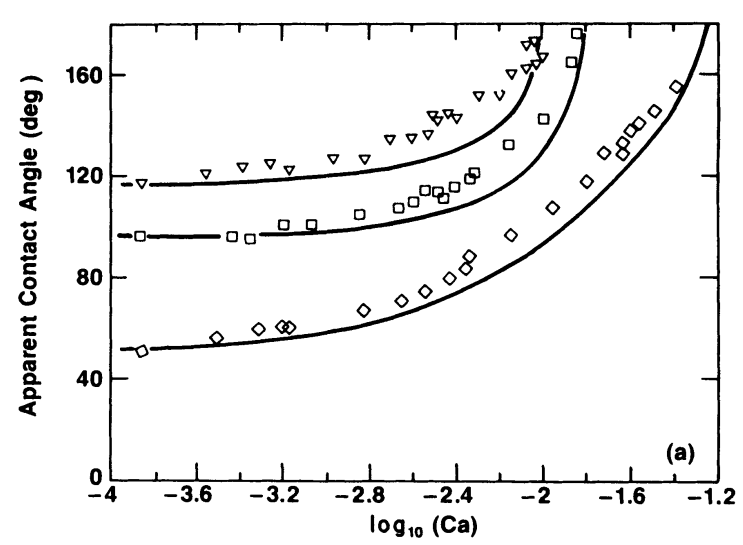

where we use the data values for $\theta$ and $\mathrm{Ca}$, and $K=0.3$. We have included the $\mathrm{Ca} \ln \mathrm{Ca}$ term in anticipation of our later analysis of the data in terms of Jansons's picture. By solving the nonlinear Eq. (11b) for $\theta_{0}$ with the appropriate value of $q$ for each data set numerically, we plot $\log _{10}\left[\cos \theta_{0}(0)-\cos \theta_{0}(\mathrm{Ca})\right]$ vs $\log _{10} \mathrm{Ca}$ in Fig. 7(b), where $\theta_{0}(0)$ and $l_{d}$ have both been varied to obtain the best linear fit to the data. The fits are very insensitive to the value of $l_{d}$, but a value of $10^{-4}$, i.e., $5000 \AA$ (for $R=0.5 \mathrm{~mm}$ ), seems to be the optimal. In any case, it is clear that Fig. 7(b) shows that the data supports the $\mathrm{Ca}$ dependence of $F$ in the form of

$$
\frac{F}{\gamma}=B \mathrm{Ca}^{x} \text {, }
$$

with $x \leq 0.5$. What we have seen, therefore, is that subtracting off the linear viscous effect makes the exponent

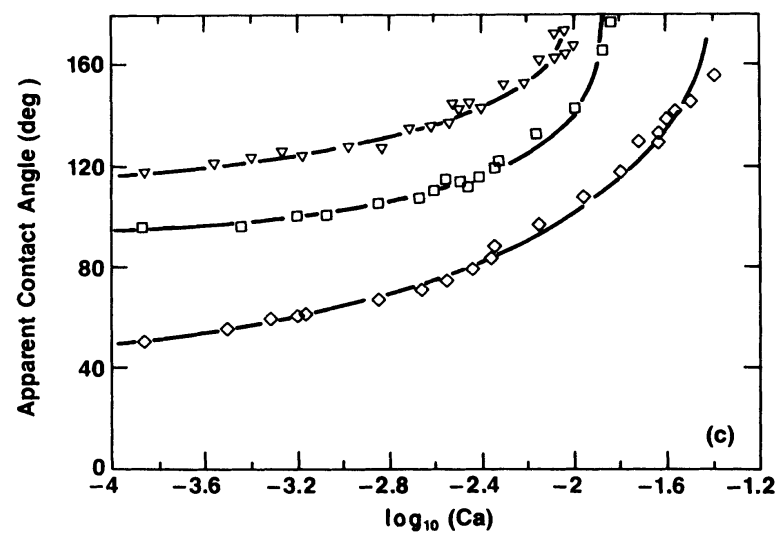

FIG. 7. Apparent contact angle plotted as a function of Ca, defined as $\eta_{1} U / \gamma$. (a) Data of Fermigier and Jenffer (Ref. [13]) measured in tubes with $R=0.5 \mathrm{~mm}$. Triangles denote a system with $\eta_{2} / \eta_{1}=0.01$, squares denote a system with $\eta_{2} / \eta_{1}=0.9$, and diamonds denote a system with $\eta_{2} / \eta_{1}-7.6 \times 10^{-3}$. The solid lines are calculated from Eqs. (6) and (7) with the appropriate $q=\eta_{2} / \eta_{1}$ values, $K=0.3$, and $l_{s}=10^{-18}, 10^{-7}$, and $10^{-19} \mathrm{~cm}$ for the triangles, squares, and the diamonds, respectively. (b) By using Eq. (11b) and the same data sets as in (a), the values of $\theta_{0}$ are calculated as a function of $\mathrm{Ca}$, with $l_{d}=5000 \AA$ and the appropriate $q$ values for each data set. What is shown are plots of $\log _{10}\left[\cos \theta_{0}(0)-\cos \theta_{0}(\mathrm{Ca})\right]$ vs $\log _{10} \mathrm{Ca}$, where $\theta_{0}(0)=105^{\circ}, 90^{\circ}$, and $40^{\circ}$ for the squares, triangles, and diamonds, respectively. For clarity, the data and the straight lines for the triangles and the diamonds have been displaced vertically by dividing by 10 and $\sqrt{10}$, respectively. The fits to the data sets yield $B=2.5$ and $x=0.29 \pm 0.01$ for the squares, $B=7.2$ and $x=0.46 \pm 0.01$ for the diamonds, and $B=4.24$ and $x=0.43 \pm 0.02$ for the triangles. (c) The data are the same as in (a). The solid lines represent Eq. (6b) evaluated with a Ca-dependent $\theta_{0}$. The parameters $B$ and $x$ for evaluating $\theta_{0}$ are obtained from the fits shown in (b). 
value $x$ for $\cos \theta_{0}(\mathrm{Ca})$ smaller than that for the apparent contact angle. The best-fitted values of $B$ and $x$ are noted in the figure caption. In Fig. 7(c) we replot Fig. 7(b) in the same format as in Fig. 7(a). Comparison with Fig. 7(a) clearly shows an improvement.

\section{B. Capillary-wave-excitation mechanism}

What is the source of this velocity-dependent frictional force? Joanny and Robbins [9] have taken the view that the contact line always follows the undulating contour of a rough wall but the velocity of the moving contact line varies as a periodic function of time. In this picture, the dominant frictional force is viscous in nature and therefore linearly proportional to $\mathrm{Ca}$; however, the effective time-averaged frictional force can deviate from linearity in its velocity dependence. By carrying out the time averaging in different ways, they obtained $x=\frac{2}{3}$ as the minimum value of $x$. Since this is not in agreement with the experimental value of $x \lesssim \frac{1}{2}$, a different physical picture must be operative.

We propose here the capillary-wave excitation at the fluid-fluid interface by wall roughness as the source mechanism of this frictional force. Basic to this picture is the Jansons's picture that the contact line "jumps" across indentations on the wall as the contact line moves forward. During the jump the fluid-fluid interface is pulled forward by interfacial tension and retarded by fluid viscosity. The jump speed is therefore on the order of $v=\gamma / \eta$, i.e., $\mathrm{Ca} \simeq 1$. As a function of time, the end of the fluid-fluid interface thus executes a nearly periodic motion with frequency $\omega_{0} \simeq 2 \pi U / l_{d}$ in the comoving frame where the overall interface remains static. Here $l_{d}$ denotes the scale of wall roughness. If $l_{0}$ is the jump distance, then $l_{0} \lesssim l_{d}$. Figure 8(a) illustrates the relation between $l_{d}$ and $l_{0}$. If the contact line moves in the slow segment at the velocity $(1-a) U$, where $a<1$, then $l_{0} \simeq a l_{d}$.

The fact that the motion of the contact line can consist of two distinct time segments as shown in Fig. 8(a) means that besides the "apparent slip length" $l_{d} / \mathrm{Ca}$ as introduced by Jansons, there can also be dissipative effects arising from the fast-moving segment in which $\mathrm{Ca} \simeq 1$. In what follows, we hypothesize that the contact line actually slips during the jump process, and that the corresponding dissipation incurred during the jump is responsible for the additional frictional force which gives rise to a velocity-dependent $\theta_{0}$. One potential slip scenario during the jump process is that the contact line actually leaves the solid surface on one side of the indentation and reattaches itself on the other side of the indentation, leaving behind a small packet of fluid 2 in fluid 1 . In fact, such a scenario would be consistent with the description of the jump segment as having $\mathrm{Ca} \simeq 1$, because without the detachment, the large viscous stress near the contact line would necessarily retard the movement so that $\mathrm{Ca}$ would be much less than 1 as a result. Our model thus has two relevant scales: the jump-slip length $l_{d}$ and the apparent slip length $l_{d} / \mathrm{Ca}$.

Let the amplitude of damped capillary-wave excitation be denoted by $l_{0} g(s, t)$, where $s=1-r$ is the coordinate normal to the wall. From what we have described above, $g(0, t)$ should behave like a sawtooth function as shown in Fig. 8(b), where in one period there is a segment of slow motion with $\partial g(0, t) / \partial t \simeq a U / l_{0}$ and another segment of fast motion corresponding to the jump. By decomposing $g(0, t)$ into a Fourier series in terms of the basis functions $\sin \left(n \omega_{0} t\right)$, we get

$$
g(0, t)=\sum_{n=1}^{\infty} C_{n} \cos \left(n \omega_{0} t-\pi / 2\right) .
$$

Here the amplitude of the sawtooth function is always normalized to 1. By assuming that the spatial dependence is of the form $\exp \left(-i k_{n} s\right)$ for each frequency component of the excitation with frequency $n \omega_{0}$, we get

$$
g(s, t)=\sum_{n=1}^{\infty} C_{n} e^{-\alpha_{n} s} \cos \left(n \omega_{0} t-\pi / 2+\beta_{n} s\right),
$$

where

$$
k_{n}=\beta_{n}+i \alpha_{n} .
$$

The total force on the solid wall can be decomposed into two components: the viscous part and the surface-tension part, which acts only at the contact line. In the uniformsurface case, the total force, and therefore the total dissipation, is related to the apparent contact angle. In fact, we have shown that $\Delta P \simeq-\cos \theta$. However, since we are now interested on the extra dissipation due to the modu-
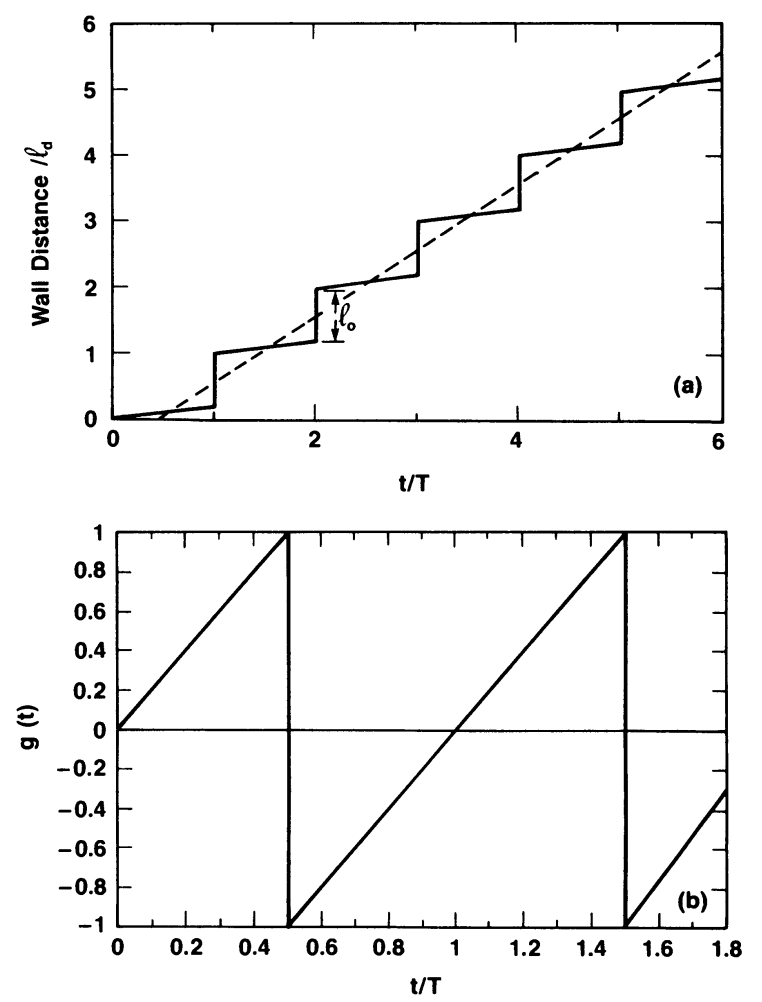

FIG. 8. (a) Contact-line motion as a function of time. The solid line denotes the actual movement. The dashed line denotes the average uniform velocity. The jump distance $l_{0}$ is noted. (b) The motion of the contact line in the comoving frame as a function of time. 
lation of $\theta_{0}$, we would like to focus on the surface-tension component of the total force. The instantaneous surface-tension part of the force along the flow direction is simply given by the instantaneous angle at which the fluid-fluid interface makes with the wall, or

$$
f \simeq-\left.\gamma \frac{\partial g(s, t)}{\partial s}\right|_{s=0}
$$

The average rate of energy dissipation $F U$ may be equated to the time average of $f l_{0} \partial g(0, t) / \partial t$ over one period. It is noted that for uniform surfaces, $\theta_{0}$ and $g(s, t)$ are constant in time and this quantity is zero. Therefore this dissipation is over and above the dissipation that is present in the uniform-surface case. Carrying out the algebra yields

$$
\frac{F}{\gamma}=a \pi l_{0} \sum_{n=1}^{\infty} n C_{n}^{2} \beta_{n} .
$$

To calculate $\beta_{n}$, one has to first obtain the dispersion relation for a damped capillary wave in the presence of a solid wall at $s=0$. In particular, we would like to have the solution for the case where the contact line is allowed to slip and therefore moves freely over $z= \pm l_{d}$, but the no-slip boundary condition applies for $z \geq\left|l_{d}\right|, s=0$. In anticipation of the result that the physically relevant solution is highly damped, we note that if the damping length in the $z$ direction is comparable to or smaller than $l_{d}$, then the no-slip boundary condition at $z \geq\left|l_{d}\right|, s=0$ essentially does not matter since the capillary-wave amplitude is negligibly small in that region anyway. That means for the physically relevant solution we can ignore the wall and just simply treat it as a free-surface problem. However, it has to be emphasized that our solution is relevant only to the jump segment of the contact-line motion where dissipation is large.

The solution to the free-surface problem has been done by Huang and Webb [17], and they obtain for the equation determining the dispersion relation

$$
S^{2}+\frac{1}{2} S\left[1+(1+2 S)^{1 / 2}\right]+y=0,
$$

where $S=i \omega \tau_{0}, \quad y=\Omega^{2} \tau_{0}^{2}, \quad \tau_{0}=\rho / 2 \eta k^{2}, \quad \Omega^{2}=\gamma k^{3} / 2 \rho$, $\rho_{1}=\rho_{2}=\rho$ being the density, and $\eta=\eta_{1}=\eta_{2}$. Derivation of the capillary-wave dispersion relation in the general case is given in the Appendix. The solution of Eq. (15) shows that for $\omega<\gamma^{2} \rho / 64 \eta^{3}=v_{0}$, the dispersion relation is of the classical form $\beta \propto \omega^{2 / 3}$. This is understandable because the capillary-wave velocity is given by $\sqrt{\gamma k / \rho}$, and therefore $\omega \propto \sqrt{k} k=k^{3 / 2}$. On the other hand, for $\omega>v_{0}$ viscous damping becomes important, and we get $\beta \propto \omega^{x}$, where $x=0.44$ at intermediate frequencies but approaches $x=0.5$ as $\omega \rightarrow \infty$. This square-root dependence corresponds to the universal diffusive nature of overdamped waves and is not limited by the condition of $\eta_{1}=\eta_{2}$ but is entirely general as shown in the Appendix. For $\gamma=30 \mathrm{dyn} / \mathrm{cm}, \rho=1 \mathrm{~g} / \mathrm{cm}^{3}$, and $\eta=0.15 \mathrm{P}$, we get $v_{0}=5 \times 10^{3} \mathrm{sec}^{-1}$. Since the dissipation is dominated by high-frequency components arising from the jump segment of the motion, the important frequency to be compared is $2 \pi v / l_{0}>2 \pi v / l_{d} \simeq 2 \pi \gamma / l_{d} \eta \simeq 10^{7}-10^{9} \mathrm{sec}^{-1}$ (for $l_{d}=1 \mu \mathrm{m}$ to $100 \AA$ ), which is much larger than $v_{0}$. Therefore, one can take the high-frequency asymptotic solution of

$$
\beta_{n}=\left(n \omega_{0} \rho / 2 \eta\right)^{1 / 2} \simeq\left(n \pi \rho U / l_{d} \eta\right)^{1 / 2} .
$$

By substituting Eq. (16) into Eq. (14), one gets

$$
\frac{F}{\gamma}=a\left(\frac{\pi^{3} \gamma \rho l_{d}}{\eta^{2}}\right)^{1 / 2}\left(\sum_{n=1}^{\infty} C_{n}^{2} n^{3 / 2}\right) \mathrm{Ca}^{1 / 2}=\bar{B} \mathrm{Ca}^{1 / 2} \text {. }
$$

It is seen immediately from the derivation above that the square-root dependence arises mainly from the diffusive nature of damped capillary waves. Since in the overdamped case $\alpha_{n}=\beta_{n}$, the damping distance in the $z$ direction (see the Appendix) is given by $\sim(\omega \rho / \eta)^{1 / 2}$ $\simeq(1-3) \times 10^{4} \mathrm{~cm}^{-1}$, where $\omega \simeq 2 \gamma / l_{d} \eta \simeq 10^{7}-10^{8} \mathrm{sec}^{-1}$ for $l_{d} \simeq 1 \mu \mathrm{m}$ to $1000 \AA$. That means the damping distance is comparable to $l_{d}$ as we supposed. In any case, the universal diffusive nature of an overdamped wave makes the result, Eq. (17), fairly general. However, the coefficient $\bar{B}$ is not completely velocity independent. This can be seen from the fact that if the ratio between the jump velocity and the slow-moving part of the velocity were to approach infinity as shown in Fig. 8(b), then the sum

$$
W=\sum_{n=1}^{\infty} C_{n}^{2} n^{3 / 2} \rightarrow \infty
$$

On the other hand, if the ratio were finite (i.e., the jump segment has $\mathrm{Ca}=1$ ) then the sum $W$ is also finite and displays a $\mathrm{Ca}^{-1 / 2}$ dependence in the limit of small $\mathrm{Ca}$. Obviously, if this were always the case then $F / \gamma$ should be velocity independent in the limit of small $\mathrm{Ca}$. However, what we have assumed above is that in one period the contact line always travels in two segments of constant speed as shown in Fig. 9(a). What is more realistic is shown in Fig. 9(b), where the transition between the two segments is not sharp but rounded due to the small but nonvanishing inertial effect. Since $W$ is a sum of $C_{n}^{2}$, its divergence behavior is sensitive to the asymptotic trend of $C_{n}$ as $n \rightarrow \infty$, i.e., the fact that whether the peak is sharp or rounded can be significant. Here the function we use is given by

$$
g(t)= \begin{cases}\sin \left(\frac{t}{1+\beta} \pi\right), & t \leq t_{c} \\ \sin \left(\frac{t-\beta}{1-\beta} \pi\right), & t>t_{c}\end{cases}
$$

for $0 \leq t \leq 1$, where $t_{c}=0.5(1-\beta)+\beta$ denotes the position of the peak, and $0 \leq \beta \leq 1$ is a parameter which shifts the peak of $g(t)$ from 0.5 at $\beta=0$ to 1 at $\beta=1$. As the ratio of the average velocities for the two segments, given by $t_{c} /\left(1-t_{c}\right)=(1+\beta) /(1-\beta)=\mathrm{Ca}$, approaches zero, $W$ still diverges, but in this case the numerical evaluation of $W$ over the relevant experimental $\mathrm{Ca}$ regime gives

$$
W \simeq 1.3 \mathrm{Ca}^{-0.12}
$$



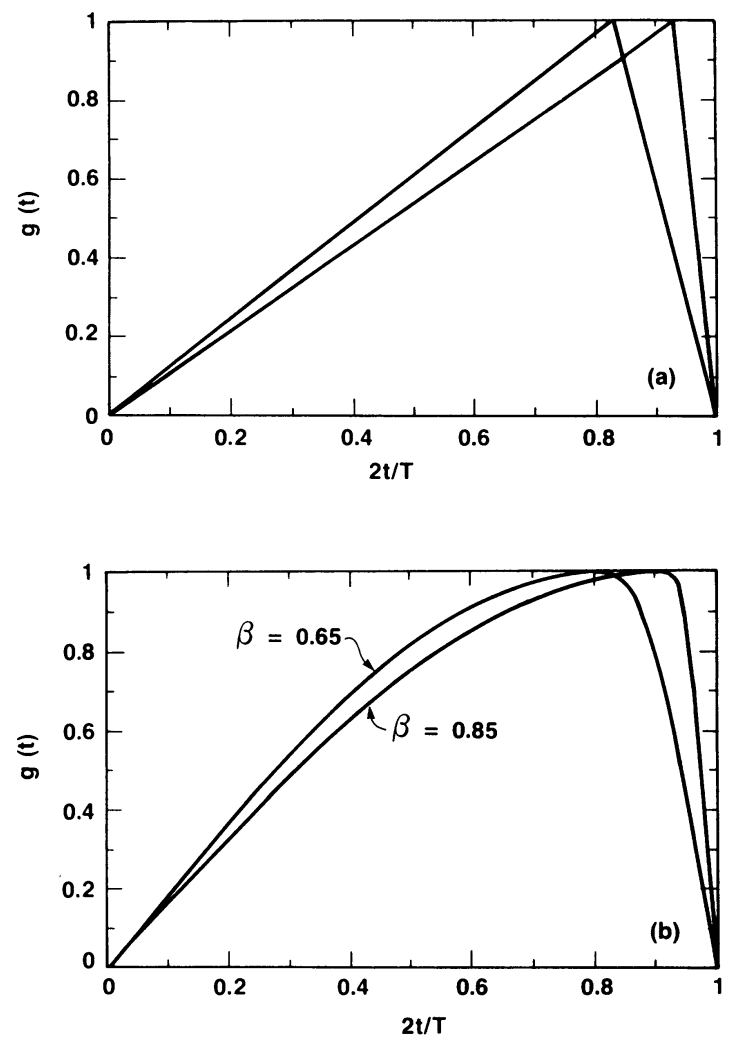

FIG. 9. (a) Contact-line motion as two segments of constant speed. In this case if the ratio of the slow velocity to the fast velocity were given by $\mathrm{Ca}$, then $W$ diverges as $\mathrm{Ca}^{-0.5}$ when $\mathrm{Ca} \rightarrow 0$. (b) The transition between the two segments is made smooth. The function $g(t)$ is given by Eq. (18a). Here $W$ diverges as $\mathrm{Ca}^{-0.12}$.

This behavior is illustrated in Fig. 10. This yields, for $g(t)$ given by Eq. (18a), a correction to the square-root behavior with the final results expressible as

$$
\frac{F}{\gamma} \simeq 1.3 a\left(\frac{\pi^{3} \gamma \rho l_{d}}{\eta^{2}}\right)^{1 / 2} \mathrm{Ca}^{0.38}=B \mathrm{Ca}^{0.38}
$$

The new coefficient $B$ is now velocity independent. For $a=1, l_{d} \simeq 1 \mu \mathrm{m}, \rho=1 \mathrm{~g} / \mathrm{cm}^{3}, \gamma=30 \mathrm{dyn} / \mathrm{cm}$, and

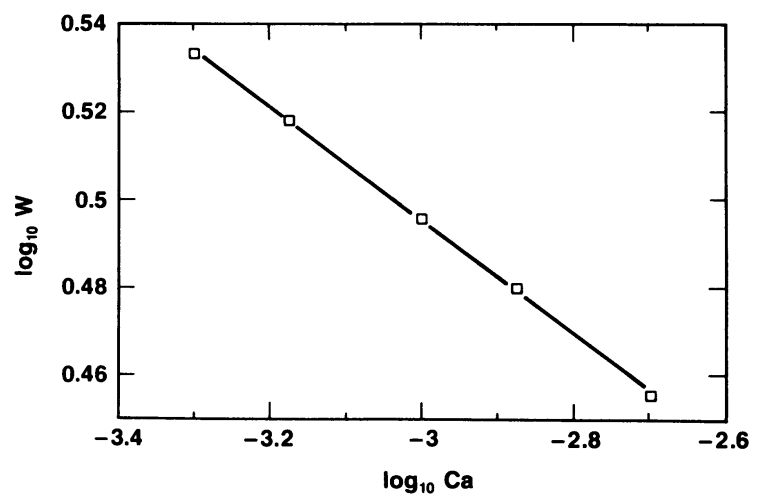

FIG. 10. Variation of $W$ as a function of $\mathrm{Ca}$, plotted in the $\log -\log$ scale. The slope of the straight line is -0.12 . $\eta=0.15 \mathrm{P}$, we get $B=2.6$. Recent accurate experiments by Stokes et al. [18] on fluid systems with $\eta_{1}=\eta_{2}$ show that $B \simeq 3.1$ and the exponent has the value of $0.4 \pm 0.05$ over the same Ca range as shown in Fig. 10. Since the $g(t)$ of Eq. (18a) is chosen arbitrarily, the agreement between theory and experiment is therefore astonishingly (or maybe fortuitously) good. Also, the assumed value of $l_{d} \simeq 1 \mu \mathrm{m}$ is noted by the same experimentalists to be within the realm of physically reasonable values for wall roughness.

What we have illustrated above is that, depending on the degree of rounding in $g(t)$, the exponent $x$ can have the value $0<x \lesssim 0.5$ over the experimentally achievable range of $10^{-4} \leq \mathrm{Ca}<10^{-2}$. As the value of $x$ decreases toward zero, the capillary-wave-generated friction becomes important only at lower and lower values of $\mathrm{Ca}$. At higher $\mathrm{Ca}$ it would appear as a near-constant and therefore difficult to separate out from the viscous friction, with $\Delta P \propto C$ a. This fact could explain the good agreement obtained between Eq. (6a), with a constant $\theta_{0}$, and some data sets [13].

A contact line moving over rough surface can excite, besides capillary waves, acoustic and solid-liquid interfacial waves as well. A valid question is why should the capillary wave be the dominant mechanism for energy dissipation at small capillary numbers. The answer may lie in the fact that compared to acoustic and Stoneley waves, which have linear dispersion relations, the capillary wave has a nonlinear dispersion that ensures its density of states to be higher than those of the other waves at small capillary numbers.

A direct prediction of our mechanism is that for solid walls smooth on the molecular scale, $B=0$ and there should only be viscous-stress effects. Also, for rough walls, the microscopic contact angle $\theta_{0}$ is noted to be a quantity which is defined only through the time-averaged force $B \mathrm{Ca}^{x}$ and Eq. (11a).

In short, our study has shown that for two-phase immiscible flow in a capillary tube, the macroscopic-flow behavior may be described by three parameters: $x, l_{d}$, and $B$. Whereas the $l_{d}$ governs the viscous effect, $x$ and $B$ control the velocity-dependent "capillary pressure." When $\eta_{1}=\eta_{2}$ and $|\cos \theta| \leq 0.6$, the excess pressure due to a moving interface is $2 \Delta P$, where $\Delta P$ may be expressed in units of $\gamma / R$ by the approximate formula

$$
\begin{aligned}
\Delta P \simeq & -\cos \theta \\
= & -\cos \theta_{0}(0)+B \mathrm{Ca}^{x} \\
& +5.63\left[\mathrm{Ca} \ln \left(K / l_{d}\right)+\mathrm{Ca} \ln \mathrm{Ca}\right],
\end{aligned}
$$

where $0<x<0.5$. Equation (20) represents the essential result of this paper. It demonstrates the connection between pressure drop, apparent contact angle, and the microscopic parameter which describe the slipping region.

\section{FREQUENCY MODULATION EFFECTS}

\section{A. Definition of the problem}

Up to now we have considered the problem of interface dynamics under the imposed condition of constant-flow 
velocity. In this section we study the effects of adding a small ac component to the imposed flow rate. The interface will now oscillate, following the imposed ac frequency $\omega$. However, as noted by Charlaix et al. [19], the important point here is that the interface can execute two types of motions. One is where the whole interface slides back and forth rigidly, and the other is the flexing, or oscillation, of the interface. Due to mass conservation, these two types of motion are coupled. Since the rigid sliding motion involves the capillary-wave generated frictional force, the ac pressure response to the imposed ac flow rate can directly yield information about the value of $x$. Indeed, this type of experimental measurements has proven to be an extremely sensitive and useful probe for nonlinear pressure-velocity relationships [18]. To relate our calculation to the experiments, in this paper we will focus our attention on the case where both $\mathrm{Ca}$ and the added ac component are small, in contrast to the case of Charlaix et al., where the ac is the only component and its amplitude need not be small. The assumption of small ac-component amplitude (relative to the dc component) enables us to obtain analytical form of the response function as is shown below.

\section{B. Mathematical formulation}

Let the imposed instantaneous flow velocity be denoted as

$$
u=U+u_{a} \exp (-i \omega t)
$$

where $U$ is the dc flow component and $u_{a}$ is the amplitude of the ac component. It is assumed that $u_{a} / U \ll 1$. In response to the imposed ac perturbation, the interface motion can consist of two components: the sliding of the contact line and the variation of the interfacial shape as parametrized by the apparent contact angle $\theta$. If the interface were rigid, then the instantaneous sliding velocity $v$ has to be equal to $u$ by mass conservation. However, the variation in the interfacial shape implies that $v$ can differ from $u$. That is, $v$ is related to $u$ by

$$
\pi R^{2} v+\frac{d V(\theta)}{d t}=\pi R^{2} u
$$

where

$$
V(\theta)=\frac{\pi R^{3}}{3} \frac{(2+\sin \theta)}{(1+\sin \theta)^{2}} \cos \theta
$$

is the volume of a spherical cap with contact angle $\theta$ as shown in Fig. 1. Direct differentiation of $V(\theta)$ yields

$$
\begin{aligned}
& \widetilde{v}+b(\theta) \frac{d \theta}{d t}=\tilde{u}, \\
& b(\theta)=-\frac{1}{(1+\sin \theta)^{2}},
\end{aligned}
$$

where $\widetilde{v}$ and $\tilde{u}$ are dimensionless velocities expressed in units of $\gamma / \eta$, and the time unit here is $\eta R / \gamma$. In addition to mass conservation, the instantaneous sliding velocity $\widetilde{v}$ is related to the contact angle $\theta$ through Eq. (20)

$$
B^{1 / x} \widetilde{v}=\left[\cos \theta_{0}(0)-\cos \theta\right]^{1 / x},
$$

where the terms $\widetilde{v} \ln \left(K / l_{d}\right)$ and $\widetilde{v} \ln \widetilde{v}$ are neglected be- cause we have assumed $\mathrm{Ca}=\eta U / \gamma$ to be small. It will be seen from the following that $\widetilde{v} \simeq \mathrm{Ca}$ to first order. Therefore $\widetilde{v}$ must also be small so that the linear term is negligible compared to the term $\widetilde{v}^{x}$, with $x \simeq 0.4$ as seen experimentally.

Equations (23) and (24) are the main equations for determining the frequency-modulation effects. To proceed further, we write

$$
\begin{aligned}
& \widetilde{v}=\widetilde{V}+\widetilde{v}_{a} \exp (-i \omega t), \\
& \theta=\theta_{\mathrm{dc}}+\theta_{a} \exp (-i \omega t) .
\end{aligned}
$$

Equation (21) can also be cast in the dimensionless form as

$$
\tilde{u}=\mathrm{Ca}+\tilde{u}_{a} \exp (-i \omega t) .
$$

By substituting Eq. (25) into Eqs. (23) and (24) and treating $\widetilde{v}_{a}, \theta_{a}$, and $\widetilde{u}_{a}$ as small perturbations, one gets from Eq. (24)

$$
\begin{aligned}
& \mathrm{Ca}=B^{-1 / x}\left[\cos \theta(0)-\cos \theta_{\mathrm{dc}}\right]^{1 / x}, \\
& \widetilde{v}_{a}=a \theta_{a}, \\
& a=\frac{1}{x} B^{-1} \mathrm{Ca}^{1-x} \sin \theta_{\mathrm{dc}} .
\end{aligned}
$$

Similar perturbation expansion from Eq. (23) yields

$$
\begin{aligned}
& \widetilde{V}=\mathrm{Ca}, \\
& \widetilde{v}_{a}-i \omega b\left(\theta_{\mathrm{dc}}\right) \theta_{a}=\tilde{u}_{a} .
\end{aligned}
$$

By using Eq. (26b), we get from Eq. (27b)

$$
\widetilde{v}_{a}=\frac{a}{a-i \omega b\left(\theta_{\mathrm{dc}}\right)} \widetilde{u}_{a} .
$$

Now from Eqs. (20) and (24), we have

$$
\begin{aligned}
\Delta P & =B \widetilde{v}^{x}-\cos \theta_{0}(0) \\
& =B\left[\mathrm{Ca}+\widetilde{v}_{a} \exp (-i \omega t)\right]^{x}-\cos \theta_{0}(0) \\
& =B \mathrm{Ca}^{x}-\cos \theta_{0}(0)+\left.\frac{\partial \Delta P}{\partial(\mathrm{Ca})}\right|_{\tilde{v}_{a}=0} \widetilde{v}_{a} e^{-i \omega t}+\cdots \\
& =\Delta P_{0}+\Delta P_{1} e^{-i \omega t}+\Delta P_{2} e^{-i 2 \omega t}+\cdots, \\
\Delta P_{0} & =B \mathrm{Ca}^{x}-\cos \theta_{0}(0), \\
\Delta P_{1} & =\left.\frac{\partial \Delta P}{\partial(\mathrm{Ca})}\right|_{\widetilde{v}_{a}=0} \widetilde{v}_{a}=x B \mathrm{Ca}^{x-1} \frac{a}{a-i \omega b\left(\theta_{\mathrm{dc}}\right)} \widetilde{u}_{a} .
\end{aligned}
$$

Equation (29) expresses the basic idea of using frequency modulation to measure $\partial(\Delta P) / \partial(\mathrm{Ca})$ (and higher derivatives) by locking into the modulation frequency (and its higher harmonics) of the pressure response. If $\Delta P$ depends nonlinearly on $\mathrm{Ca}$, then by measuring its derivatives one can accurately determine its nonlinear exponent $x$. However, due to the effect of interfacial shape modulation, $\tilde{u}_{a}$ can differ from $\widetilde{v}_{a}$ as seen from Eq. (28). As a result, the sliding component of the response can be masked by considerable frequency dependence. 


\section{Frequency dependence of the pressure response}

We will focus our attention on $\Delta P_{1}$, the pressure response at frequency $\omega$. By using Eq. (26c), Eq. (29c) can be expressed as

$$
\frac{\Delta P_{1}}{\widetilde{u}_{a}}=\frac{\sin \theta_{\mathrm{dc}}}{x^{-1} B^{-1} \sin \theta_{\mathrm{dc}} \mathrm{Ca}^{1-x}+i \omega\left(1+\sin \theta_{\mathrm{dc}}\right)^{-2}},
$$

where we have normalized our pressure response to the impose perturbation amplitude. In the limit of $\omega \rightarrow 0$, we get

$$
\begin{aligned}
& \lim _{\omega \rightarrow 0} \operatorname{Re}\left(\frac{\Delta P_{1}}{\widetilde{u}_{a}}\right)= x B \mathrm{Ca}^{x-1} \\
& \lim _{\omega \rightarrow 0} \operatorname{Im}\left(\frac{\Delta P_{1}}{\widetilde{u}_{a}}\right)=- \omega x^{2} B^{2}\left(\sin \theta_{\mathrm{dc}}\right)^{-1}\left(1+\sin \theta_{\mathrm{dc}}\right)^{-2} \\
& \times \mathrm{Ca}^{2 x-2}
\end{aligned}
$$

It is seen that the real part should be much larger than the imaginary part and contains the $\mathrm{Ca}$ dependence that arises from rigid sliding of the contact line. On the other hand, for $\omega \rightarrow \infty$ we have

$$
\begin{aligned}
\lim _{\omega \rightarrow \infty} \operatorname{Re}\left(\frac{\Delta P_{1}}{\widetilde{u}_{a}}\right)= & \omega^{-2} x^{-1} B^{-1} \sin ^{2} \theta_{\mathrm{dc}}\left(1+\sin \theta_{\mathrm{dc}}\right)^{4} \\
& \times \mathrm{Ca}^{1-x} \\
\lim _{\omega \rightarrow \infty} \operatorname{Im}\left(\frac{\Delta P_{1}}{\widetilde{u}_{a}}\right)= & -\omega^{-1}\left(1+\sin \theta_{\mathrm{dc}}\right)^{2} \sin \theta_{\mathrm{dc}}
\end{aligned}
$$

Now the real part is much smaller than the imaginary part, and its Ca dependence is inverse to that of the lowfrequency dependence (if we discount the weak Ca dependence of $\sin \theta_{\mathrm{dc}}$ ). The high-frequency behavior reflects the physical process of interfacial oscillation with a pinned contact line.

The frequency that separates the low-frequency regime from the high-frequency regime is obvious from the denominator of Eq. (30), i.e.,

$$
\omega_{c}=x^{-1} B^{-1} C^{1-x} \sin \theta_{d c}\left(1+\sin \theta_{d c}\right)^{2} .
$$

For a given $\mathrm{Ca}, \omega<<\omega_{c}$ means the low-frequency regime. If we let $\mathrm{Ca}=10^{-4}, \theta_{\mathrm{dc}} \simeq 90^{\circ}, x=0.4, B=3$, then $\omega_{c} \simeq 0.01$. In terms of the actual time units $\eta R / \gamma$, this translates into a frequency of $\sim 3 \mathrm{~Hz}$ for $\gamma=30 \mathrm{dyn} / \mathrm{cm}$, $\eta=0.15 \mathrm{P}$, and $R=0.1 \mathrm{~cm}$. That is, for $\omega / 2 \pi<3 \mathrm{~Hz}$ we can expect the $\mathrm{Ca}$ dependence to follow Eq. (31a) for $\mathrm{Ca}>10^{-4}$. This critical frequency is in good agreement with what has actually been observed by Stokes et al. [20]. Above $\omega_{c}$ the oscillation of the interface introduces considerable imaginary part to the response, and the $\mathrm{Ca}$ dependence is expected to deviate significantly from Eq. (31a). In Fig. 11 we plot the Ca dependence of $\left(\Delta P_{1} / \tilde{u}_{a}\right)$ as calculated from Eqs. (30) and the relation

$$
\theta_{\mathrm{dc}}=\cos ^{-1}\left[\cos \theta_{0}(0)-B \mathrm{Ca}^{x}\right] \text {, }
$$

obtained from Eq. (24). It is seen that for $\omega=0.01$ the real part of $\left(\Delta P_{1} / \tilde{u}_{a}\right)$ follows the low-frequency behavior
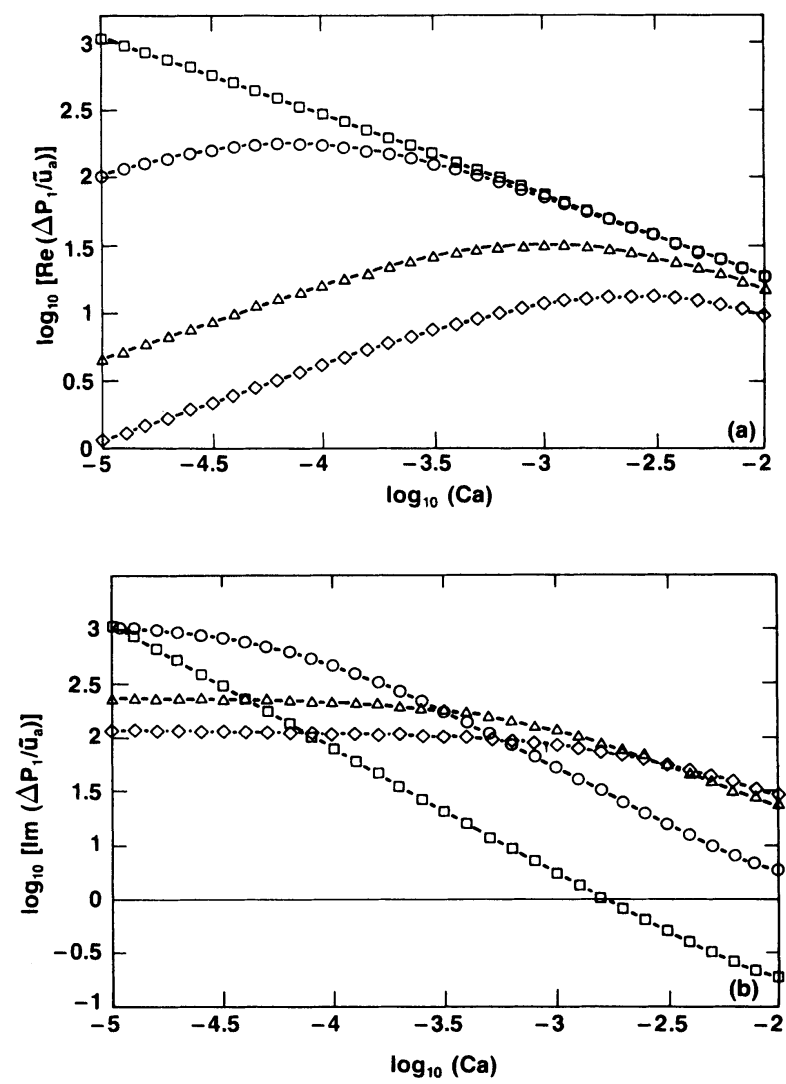

FIG. 11. Dependence of $\Delta P / \widetilde{u}_{a}$ on the capillary number for several frequencies, calculated with $\theta_{\mathrm{dc}}=105^{\circ}, B=3$, and $x=0.4$. In units of $\gamma / R \eta$, the curves for different angular frequencies are denoted by different symbols: open square, $\omega=0.001$; open circle, $\omega=0.01$; open diamond, $\omega=0.1$; and open triangle, $\omega=0.05$. (a) Real part. (b) Imaginary part. Here $\Delta P_{1}$ is in units of $\gamma / R$ and $\tilde{u}_{a}$ is in units of $\gamma / \eta$. For $\omega=0.001$ the real part is seen to be a straight line with the slope of -0.6 .

given by Eq. (31a) for $\mathrm{Ca}>10^{-4}$. Experimentally, precisely those behaviors as shown in Fig. 11 were measured [20].

One implication of the above result is that, since the capillary-wave-generated frictional force is much larger than the viscous friction at low capillary numbers, the rigid sliding part of the ac motion can introduce additional dissipation for low-frequency waves traveling through porous media. This could be the explanation for extra dissipation observed for seismic waves in partially saturated rocks [21].

\section{SUMMARY AND CONCLUDING REMARKS}

We have shown that both the shape of the interface and the macroscopic immiscible-flow behavior are determined by the microscopic parameters governing the contact-line region. Two dominant frictional forces have been identified. One is the viscous stress generated in the slipping region, which is responsible for the deformation of the fluid-fluid interface and the deviation of the apparent contact angle from the microscopic contact angle. 
The other friction, due to the damped capillary-wave excitations at the fluid-fluid interface, is shown to induce a nonlinear velocity dependence on the microscopic contact angle. These effects are describable in terms of the microscopic parameters as expressed by Eq. (20). Frequency-modulation effects have been deduced from these relations by the additional condition of mass conservation.

While progress has been made in understanding immiscible-flow behavior, it is noted that the present model involving rough-wall surfaces relies on assumed behaviors of contact-line "jumps" across indentations. Obviously, there is a need for making the plausible assumptions more rigorous. This will be the task of future investigations.

\section{ACKNOWLEDGMENTS}

The authors wish to acknowledge helpful discussions with J. P. Stokes, E. Charlaix, and M. Robbins.

\section{APPENDIX}

In this appendix we derive the general dispersion relation for the damped capillary wave at a flat interface. Figure 12 defines the relevant coordinates and parameters of the problem. Here the interface is defined to be $z=0$. The parameters related to the fluid at $z>0$ are denoted by subscript 2 , i.e., $p_{2}, \eta_{2}, \rho_{2}$, etc., and those related to the fluid at $z<0$ are denoted by subscript 1 , i.e, $p_{1}, \eta_{1}, \rho_{1}$, etc.

The general linearized equation of motion for incompressible fluid is

$$
\begin{aligned}
\frac{\partial}{\partial t} \mathbf{v} & =\frac{\eta}{\rho} \nabla^{2} \mathbf{v}-\frac{1}{\rho} \nabla p \\
\boldsymbol{\nabla} \cdot \mathbf{v} & =0 .
\end{aligned}
$$

For interfacial excitations, the boundary conditions are

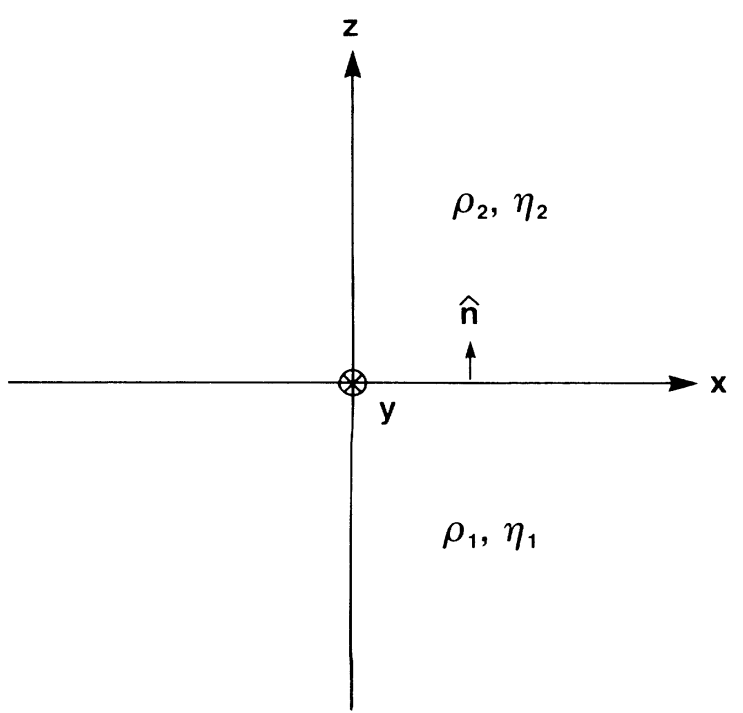

FIG. 12. Coordinates and material parameters for calculating the dispersion relation of damped capillary waves.

$$
\begin{aligned}
& \mathbf{v}_{2}, \rho_{2} \rightarrow 0 \text { for } z \rightarrow \infty, \\
& \mathbf{v}_{1}, \rho_{1} \rightarrow 0 \text { for } z \rightarrow-\infty, \\
& \mathbf{v}_{1}=\mathbf{v}_{2} \text { at } z=0, \\
& \left(p_{1}-p_{2}\right) \hat{\mathbf{n}}=\left(\sigma^{(1)}-\sigma^{(2)}\right) \cdot \hat{\mathbf{n}}+2 \gamma \kappa \hat{\mathbf{n}} \text { at } z=0 .
\end{aligned}
$$

Here the last condition is exactly that of Eq. (2a), except here we have not nondimensionalized the variables.

To proceed, we write down the general forms for $\mathbf{v}_{1}$ and $\mathbf{v}_{2}$,

$v_{1 x}=\exp [i(k x-\omega t)]\left[A \exp (k z)+B \exp \left(m_{1} z\right)\right]$,

$v_{l y}=0$

$v_{2 x}=\exp [i(k x-\omega t)]\left[C \exp (-k z)+D \exp \left(-m_{2} z\right)\right]$,

$v_{2 y}=0$.

Here the presence of the terms $\exp \left(m_{1} z\right)$ and $\exp \left(-m_{2} z\right)$ are required to accommodate viscous losses as seen below. Given $v_{x}$ and $v_{y}, v_{z}$ can be obtained from the incompressibility condition, Eq. (A2)

$$
\begin{aligned}
v_{1 z}=-i \exp [i(k x-\omega t)] & A \exp (k z) \\
& \left.+B \frac{k}{m_{1}} \exp \left(m_{1} z\right)\right),
\end{aligned}
$$

$$
\begin{aligned}
v_{2 z}= & i \exp [i(k x-\omega t)] \\
& \times\left(C \exp (-k z)+D \frac{k}{m_{2}} \exp \left(-m_{2} z\right)\right) .
\end{aligned}
$$

By taking the curl of Eq. (A1), one can get rid of $\nabla p$ and obtain a relation between $m_{1,2}$ and $k$

$$
\begin{aligned}
& m_{1}^{2}=k^{2}-i \omega \rho_{1} / \eta_{1}, \\
& m_{2}^{2}=k^{2}-i \omega \rho_{2} / \eta_{2} .
\end{aligned}
$$

By again returning to Eq. (A1), we obtain pressure at $z=0$ as

$$
\begin{aligned}
& p_{1}(z=0)=\frac{\rho_{1} \omega}{k} A, \\
& p_{2}(z=0)=\frac{\rho_{2} \omega}{k} C .
\end{aligned}
$$

Now we have all the ingredients for matching boundary conditions. From Eq. (A5), we obtain

$$
\begin{aligned}
& A+B-C-D=0 \\
& A+\frac{k}{m_{1}} B+C+\frac{k}{m_{2}} D=0 .
\end{aligned}
$$


From Eq. (A6), we get

$$
\begin{array}{r}
\left(\frac{\gamma k^{2}}{\omega}-\frac{\rho_{1} \omega}{k}-2 i \eta_{1} k\right) A+\left(\frac{\gamma k^{3}}{\omega m_{1}}-2 i k \eta_{1}\right) B \\
-\left(\frac{\rho_{2} \omega}{k}-2 i k \eta_{2}\right) C+2 i k \eta_{2} D=0,
\end{array}
$$

$$
\begin{gathered}
2 \eta_{1} k A+\eta_{1}\left(m_{1}+\frac{k^{2}}{m_{1}}\right) B+2 \eta_{2} k C+\eta_{2}\left(m_{2}+\frac{k^{2}}{m_{2}}\right) D \\
=0 . \quad \text { (A20) }
\end{gathered}
$$

The dispersion relation can be obtained by simply requiring the coefficient matrix to have a zero determinant. By using Eq. (A17) to eliminate $C$, we get a $3 \times 3$ determinant as

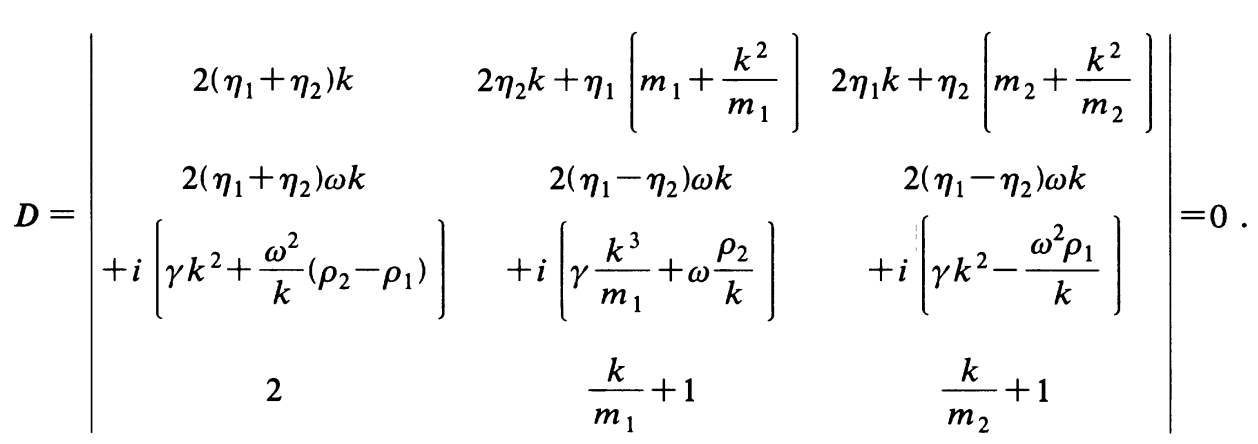

By defining $q=\eta_{2} / \eta_{1}, \bar{\rho}=\rho_{2} / \rho_{1}$,

$$
\begin{aligned}
& S=-i \frac{\omega \rho_{1}}{2 \eta_{1} k^{2}}, \\
& y=\frac{\rho_{1} \gamma}{8 \eta_{1}^{2} k},
\end{aligned}
$$

we get

$$
\begin{aligned}
& m_{1}=\sqrt{1+2 S} k=\sigma_{1} k, \\
& m_{2}=\frac{\sqrt{1+2 \bar{\rho} S}}{q} k=\sigma_{2} k,
\end{aligned}
$$

and the determinant becomes

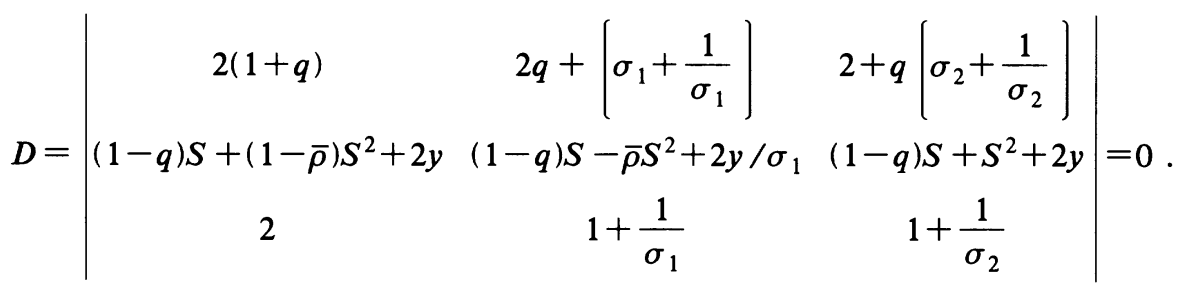

In the limit of $\omega \rightarrow \infty$, let us try the ansatz $\omega \propto k^{2}$. In that case $y \rightarrow 0$ and $S$ should be a material constant. From Eq. (A26) it is easily seen that this is indeed the case. Therefore, in general

$$
\lim _{\omega \rightarrow \infty} k \propto \sqrt{\omega},
$$

independent of material parameters. The fact that this is the case reflects the general diffusive nature of an overdamped wave.

For the case $q=1, \bar{\rho}=1$, we get $\sigma_{1}=\sigma_{2}=\sigma$, and

$$
D=\left|\begin{array}{ccc}
2 & 2+\left(\sigma+\frac{1}{\sigma}\right) & 2+\left(\sigma+\frac{1}{\sigma}\right) \\
y & \frac{2 y}{\sigma}-S^{2} & 2 y+S^{2} \\
1 & 1+\frac{1}{\sigma} & 1+\frac{1}{\sigma}
\end{array}\right|=0
$$


From Eq. (A27) we immediately get

$$
S^{2}+\left(1-\frac{1}{\sigma}\right) y=0
$$

By using the fact that $\sigma=\sqrt{1+2 S}$, one recovers Eq. (15).

[1] E. B. Dussan, V., Ann. Rev. Fluid Mech. 11, 371 (1979).

[2] P. G. DeGennes, Rev. Mod. Phys. 57, 827 (1985).

[3] L. M. Hocking, J. Fluid Mech. 76, 801 (1976); 79, 209 (1977).

[4] J. Koplik, J. R. Banavar, and J. F. Willemsen, Phys. Rev. Lett. 60, 1282 (1988).

[5] P. A. Thompson and M. O. Robbins, Phys. Rev. Lett. 63, 766 (1989).

[6] M. Y. Zhou and P. Sheng, Phys. Rev. Lett. 64, 882 (1990).

[7] C. Huh and S. C. Mason, J. Fluid Mech. 81, 401 (1977).

[8] K. M. Jansons, J. Fluid Mech. 154, 1 (1985); 167, 393 (1986).

[9] J. F. Joanny and M. O. Robbins, J. Chem. Phys. 92, 3206 (1990); E. Raphael and P. G. de Gennes, ibid. 90, 7577 (1989).

[10] R. L. Hoffman, J. Colloid Interface Sci. 50, 228 (1975).

[11] R. G. Cox, J. Fluid Mech. 168, 169 (1986).

[12] E. B. Dussan, V. and S. H. Davis, J. Fluid Mech. 173, 115 (1986).
[13] G. M. Fermigier and P. Jenffer, Ann. Phys. (Paris) 13, 37 (1988).

[14] P. A. Thompson and M. O. Robbins, Phys. World 3, 35 (1990).

[15] M. Fermigier, doctoral thesis, Université Pierre et Marie Curie, 1989.

[16] T. E. Mumley, C. J. Radke, and M. C. Williams, J. Colloid Interface Sci. 109, 398 (1986).

[17] J. S. Huang and W. W. Webb, Phys. Rev. Lett. 23, 1650 (1969).

[18] J. P. Stokes et al., Phys. Rev. Lett. 65, 1885 (1990).

[19] E. F. Charlaix et al., (unpublished); J. P. Stokes, E. F. Charlaix, A. P. Kushnick, and S. Bhattacharya, in Fractal Aspects of Materials: Disordered Systems, Materials Research Society Extended Abstracts, edited by D. A. Weitz, L. M. Sander, and B. B. Mandelbrot (MRS, Pittsburgh, (1988), p. 91.

[20] J. P. Stokes (private communication).

[21] M. J. Miksis, J. Geo. Res. 93, 6624 (1988). 